\title{
Effect of Mediterranean diet versus prudent diet combined with physical activity on OSAS: a randomised trial
}

\author{
Christopher Papandreou*, Sophia E. Schiza\#, Izolde Bouloukaki ${ }^{\#}$, Christos \\ M. Hatzis*, Anthony G. Kafatos*, Nikolaos M. Siafakas" and Nikolaos E. Tzanakis ${ }^{\#, \text {, }}$
}

ABSTRACT: We aimed to evaluate the effect of the Mediterranean diet (MD) compared with a prudent diet (PD) combined with physical activity on obese obstructive sleep apnoea syndrome (OSAS) patients who were treated with continuous positive airway pressure.

900 patients were evaluated and 40 obese patients (body mass index $\geqslant 30.0 \mathrm{~kg} \cdot \mathrm{m}^{-2}$ ) who met the inclusion criteria, with moderate-to-severe OSAS (apnoea-hypopnoea index (AHI) >15 events $\cdot \mathrm{h}^{-1}$ and Epworth Sleepiness Scale score $>10$ ) based on overnight attended polysomnography, were included in the study. After randomisation, 20 patients followed the MD and 20 a PD for a 6-month period. All patients were counselled to increase their physical activity.

Concerning sleep parameters, only AHI during rapid eye movement (REM) sleep was reduced to a statistically significant degree, by mean \pm SD $18.4 \pm 17.6$ events $\cdot h^{-1}$ in the MD group and by $2.6 \pm 23.7$ events $\cdot h^{-1}$ in the PD group $(p<0.05)$. The MD group also showed a greater reduction in waist circumference (WC) $(-8.7 \pm 3.6 \mathrm{~cm})$, WC/height ratio $\left(-0.04 \pm 0.02 \mathrm{~cm} \cdot \mathrm{m}^{-1}\right)$ and WC/hip ratio $\left(-0.04 \pm 0.03 \mathrm{~cm} \cdot \mathrm{cm}^{-1}\right)$, compared with the other group $\left(-2.6 \pm 1.7\right.$ events $\cdot \mathrm{h}^{-1},-5.7 \pm 3.8 \mathrm{~cm}$, $-0.03 \pm 0.02 \mathrm{~cm} \cdot \mathrm{m}^{-1}$ and $0.02 \pm 0.02 \mathrm{~cm} \cdot \mathrm{cm}^{-1}$, respectively; $\left.\mathrm{p}<0.05\right)$.

Our results showed that the MD combined with physical activity for a 6-month period was effective in reducing the AHI during REM sleep without any statistically significant effect in the other sleep parameters, compared with a PD in obese adults with moderate-to-severe OSAS.

KEYWORDS: Abdominal obesity, apnoea-hypopnoea index, Mediterranean diet, obstructive sleep apnoea syndrome, physical activity, rapid eye movement sleep

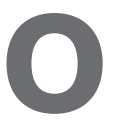
bstructive sleep apnoea syndrome (OSAS) is considered to be one of the most prevalent sleep-related breathing disorders, with an enormous effect on public health. Approximately $2-4 \%$ of the general adult population experiences some degree of this syndrome [1]. This percentage increases even more with obesity, up to $20-40 \%$, especially in individuals with an excessive body mass index (BMI) $\left(\geqslant 30 \mathrm{~kg} \cdot \mathrm{m}^{-2}\right)$ [2]. OSAS is associated with significant systemic consequences, including cardiovascular morbidity and mortality, and the risk increases with the severity of the syndrome [3]. The pathophysiology underlying the link between OSAS and the cardiovascular system is attributed largely to systemic inflammation and oxidative stress, which are both contributors to endothelial dysfunction [4]. The treatment modalities of OSAS include continuous positive airway pressure (CPAP), weight loss, upper airway surgery and oral devices [5]. CPAP is the first-line therapy, as a means of maintaining upper airway patency [6], and it is well known that this intervention reduces morbidity and mortality [7]. In severe cases of OSAS, the weight loss strategy is essential and must accompany CPAP treatment [8]. A growing body of evidence in OSAS supports the beneficial role of weight reduction, induced by diet alone [9-12] or in combination with exercise [13], leading to a reduction in apnoeas and hypopnoeas. However, randomised controlled trials examining this issue are rare, while available observational studies suffer from several biases and confounders, and their data are limited [14]. To the best of our knowledge, only two randomised studies inducing weight loss have been reported so far. Both observed a clinically meaningful improvement in patients with mild [12] and moderate-to-severe [11] OSAS. Most of the weight reduction and sleep apnoea studies did not include

\section{AFFILIATIONS}

*Dept of Social Medicine, Preventive Medicine and Nutrition Clinic ${ }^{\#}$ Dept of Thoracic Medicine, Sleep Disorders Unit, University General Hospital, and

"Dept of Epidemiology, Social Medicine, Medical School of the University of Crete, Heraklion, Greece.

CORRESPONDENCE

C. Papandreou

Preventive Medicine and Nutrition

Clinic, University of Crete

School of Medicine

POB 2208 GR-71003

Heraklion

Crete

Greece

E-mail: papchris10@gmail.com

Received:

June 162011

Accepted after revision:

Sept 072011

First published online:

Oct 272011 
exercise in their intervention programme. Exercise combined with a change in dietary behaviour has been shown to result in greater weight loss [15]. Moreover, these studies evaluated the effects of low- and very low-calorie diets on obese patients with OSAS. It was suggested recently that the Mediterranean diet (MD) is beneficial for weight loss [16], but its role in obese OSAS patients has never been explored. The MD promotes satiation and encourages consumption of a variety of palatable foods, optimising adherence to a calorie-restricted diet [17]. This dietary pattern would result in greater weight loss and, hence, greater improvement in OSAS compared with a general weight reduction diet.

Therefore, we aimed to evaluate the effect of the MD compared with that of a prudent diet (PD) on obese OSAS patients who were treated with CPAP while receiving counselling to increase their physical activity.

\section{METHODS}

\section{Participants}

A series of consecutive patients, who were diagnosed with OSAS by overnight attended polysomnography (PSG) at the Sleep Disorders Unit, Dept of Thoracic Medicine, Medical School, University of Crete (Heraklion, Greece), during a 1-yr period (November 2008 to October 2009) were evaluated and the study population was selected based on the following criteria. Inclusion criteria were: 1) age $18-65 \mathrm{yrs}$; 2) $\mathrm{BMI} \geqslant 30$ $\mathrm{kg} \cdot \mathrm{m}^{-2}$; and 3) apnoea-hypopnoea index (AHI) $>15$ events $\cdot \mathrm{h}^{-1}$. Exclusion criteria were: 1) diseases such as cardiac ischaemic disease, diabetes mellitus, thyroid disorders, psychotic disease and malignancies, and alcoholism; 2) upper airway surgery; 3) pregnancy; 4) diet for weight reduction in the last 6 months or medications affecting weight; 5) eating habits close to the MD at the entry phase; and 6) treatment with sleeping pills. The study flowchart is shown in figure 1 . After the visit to the study physicians and confirmation that the patients fulfilled the inclusion/exclusion criteria, the subjects were allocated randomly to two study groups using a computer-generated random number sequence. No stratification was used in the allocation of the participants into the two groups. The groups were matched by sex. In view of the use of both diets, blinding of staff or patients was not considered feasible. The primary outcome measure was the change in OSAS severity as reflected mainly by AHI and saturation indexes. In addition to these measurements, we recorded the AHI during rapid eye movement (REM) sleep. This study was approved by the ethical committee of the University of Crete and all the participants provided signed consent.

\section{Intervention}

Two groups of patients with moderate-to-severe OSAS were formed. In both groups, the patients received CPAP therapy and lifestyle intervention including a programme of increasing physical activity, mainly involving walking for $\geqslant 30$ min daily. At the same time, the two groups were advised to follow a lowcalorie diet, one a PD and the other a MD. In all patients, a specific motivation programme and education in reducing calorie intake were offered, aiming to restrict the daily energy to $1,200-1,500 \mathrm{kcal}(\sim 5,000-6,000 \mathrm{~kJ})$ for females and 1,500$1,800 \mathrm{kcal}(\sim 6,000-7,500 \mathrm{~kJ})$ for males. The general guidelines to the participants in the MD group were consumption of six servings per day of nonrefined cereals, five or more servings per week of potatoes, five servings per day of various vegetables (two of them as salad), four servings per day of various fresh fruits, three or more servings per week of legumes, three servings per week of fish (at least one serving of oily fish), one serving per day of nuts, three servings per week of poultry without skin, three servings per week of red meat and seven glasses each week of red wine. The recommended intake of fruits, vegetables, legumes, nonrefined cereals and fish was three times higher in the MD group than in the PD group, whereas the red meat intake in the former group was one-third of that in the latter. The moderate daily consumption of nuts and alcohol (red wine) was only recommended in the MD group. In both groups the moderate consumption of olive oil was recommended, because based on the local conditions almost all the people living on the island of Crete produce and consume this type of oil. Patients in both groups were also advised to eliminate or limit the consumption of cream, butter, margarine, carbonated and/or sugared beverages, commercial bakery products (e.g. sweet desserts, cakes, biscuits/cookies, puddings, and custard), potato fries and processed meats (i.e. burgers and sausages), and to consume two servings per day of low-fat dairy products.

The intervention lasted for 6 months, and consisted of a total of seven visits, including a dietician visit and consultation. The study dietician was responsible for providing dietary and exercise counselling at each visit. Additionally, compliance with CPAP therapy was monitored.

\section{Procedures and measurements}

Overnight attended PSG (Alice 5; Philips Respironics, Best, the Netherlands) was performed in the Sleep Disorders Unit of the University of Crete. Patients underwent a full diagnostic PSG study, according to standard techniques, with monitoring of the electroencephalogram (EEG) using frontal, central and occipital leads, electro-oculogram, electromyogram, flow (by oronasal thermistor and nasal air pressure transducer), thoracic and abdominal respiratory effort by uncalibrated impedance plethysmography belts, oximetry, and body position. Snoring was recorded by a microphone placed on the anterior neck. A single modified type II ECG lead was used for cardiac monitoring. PSG recordings were manually interpreted over 30-s periods, in accordance with the American Academy of Sleep Medicine (AASM) guidelines [18, 19]; the scorer was blinded to the PSG findings of the initial and final clinical assessment. The determination of sleep stages and arousals was performed according to the AASM 2007 criteria and by using EEG montages including frontal, central and occipital leads. Two sleep recordings were performed during the study. The first was conducted at baseline and the second at 6 months. Patients with AHI $>15$ events $\cdot h^{-1}$ and Epworth Sleepiness Scale (ESS) score $>10$ were assigned as having moderate-to-severe OSAS and underwent a CPAP titration study. After CPAP initiation, patients were followed up by the Sleep Disorders Unit CPAP clinic. The hours per day and percentage of days that CPAP was used were monitored (IC card; Philips Respironics) at the monthly clinical assessment. All patients exhibited good compliance, using CPAP $>4 \mathrm{~h}$ per day and $>5$ days per week, which are the criteria of regular use [20]. The end of the PSG 


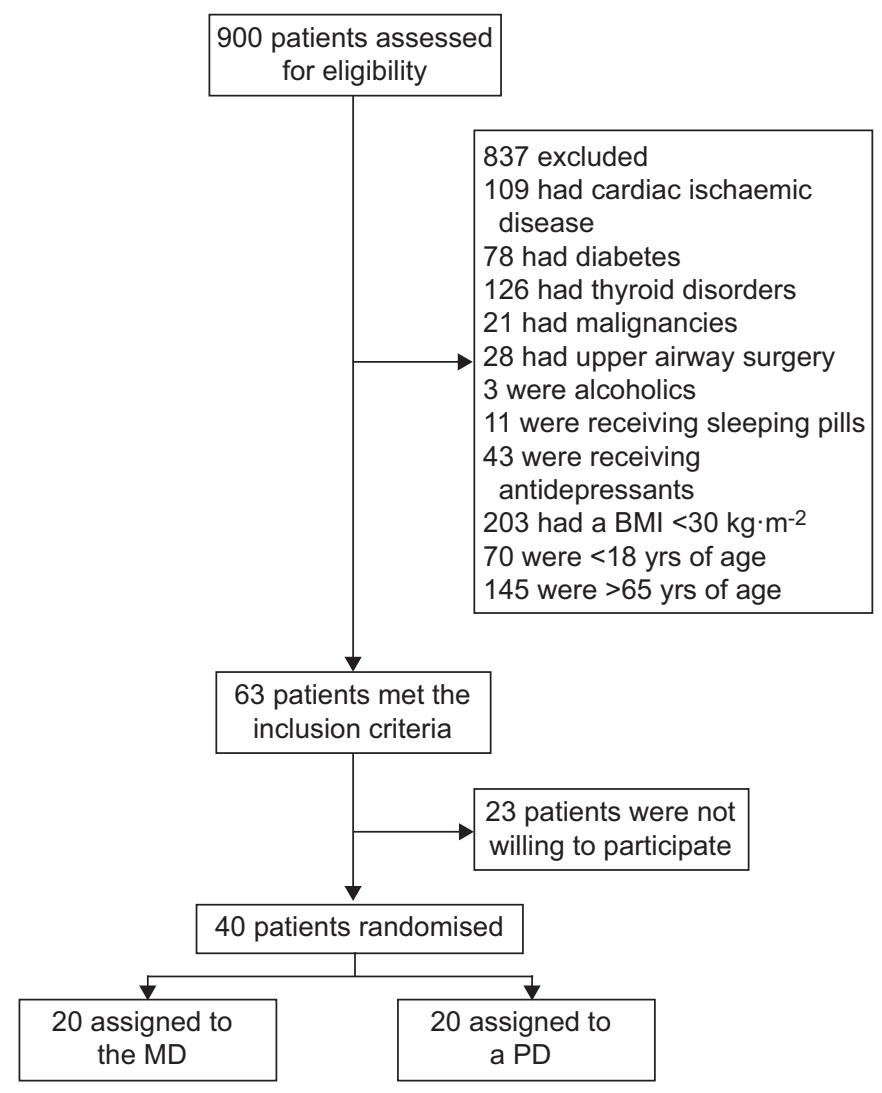

FIGURE 1. Study flowchart. A total of 40 patients were enrolled; 20 were randomly assigned to the Mediterranean diet (MD) and 20 were randomly assigned to a prudent diet (PD). BMI: body mass index.

study was performed without CPAP. One day prior to PSG, CPAP was ceased.

Anthropometric measurements (weight, height, waist circumference (WC) and neck circumference (NC)) were carried out by an expert at baseline and at each visit, and a subject was defined as obese when their BMI was $\geqslant 30.0 \mathrm{~kg} \cdot \mathrm{m}^{-2}$ [21]. Body fat was estimated by the four-skinfold thickness method [22] at baseline, and at the 6-month visit. Adherence to the diets was evaluated by a semiquantitative food frequency questionnaire [23] at baseline, at the 3-month visit and at the 6-month visit. In addition, overall dietary habits were evaluated through a special diet score (MedDietScore, range 0-55) that assessed adherence to the MD pattern [24]. Physical activity was assessed using the long version of the self-reported International Physical Activity Questionnaire [25] at baseline, at the 3-month visit and at the 6-month visit. Participants were also questioned regarding their educational level and smoking status. Educational level was categorised in four levels (0: primary school; 1: secondary school; 2: post-high school education; 3: higher education).

\section{Statistical analysis}

Mean values and standard deviations were used to describe the baseline characteristics of the two treatment groups. Unpaired t-tests were used to assess equality between the treatment groups at baseline. Changes in sleep recordings and anthropometric measurements during the follow-up period were calculated by subtracting the baseline measurement from the 6-month follow-up measurement, and the mean difference in changes between the treatment groups was calculated. The statistical significance of between-group differences in changes was assessed with unpaired t-tests. All data were analysed with the SPSS version 18.0 (IBM, Somers, NY, USA). A p-value $<0.05$ was considered significant.

\section{RESULTS}

40 patients were randomised to the PD group $(n=20)$ or MD group $(n=20)$; all of them successfully completed this small, underpowered study. No side-effects of either treatment were reported.

During the selection of the study population, 837 patients were excluded based on the abovementioned exclusion criteria (109 patients had ischaemic heart disease, 78 patients had diabetes mellitus, 126 patients had thyroid disorders, 21 patients suffered from malignancies, 28 had undergone upper airway surgery, three were alcoholics, 11 were under therapy with sleeping pills, 43 were receiving antidepressant medication, 203 had a BMI $<30.0 \mathrm{~kg} \cdot \mathrm{m}^{-2}, 70$ were $<18 \mathrm{yrs}$ of age and 145 were $>65$ yrs of age). Another 23 patients refused to participate in the intervention programme (fig. 1).

Table 1 shows the characteristics of the two groups at baseline. Despite the randomisation, BMI was higher in the PD group compared with the MD group (mean \pm SD $37.9 \pm 4.6$ versus $\left.35.3 \pm 3.6 \mathrm{~kg} \cdot \mathrm{m}^{-2} ; \mathrm{p}=0.04\right)$. There were no significant differences between the two intervention groups in the other baseline characteristics $(\mathrm{p}>0.05)$. In general, the patients studied were middle aged $(48.9 \pm 12.7 \mathrm{yrs})$; the majority were males $(85.0 \%)$, with moderate-to-severe OSAS (AHI $>15$ events $\cdot h^{-1}$ and ESS score $>10)$ and BMI $36.6 \pm 3.7 \mathrm{~kg} \cdot \mathrm{m}^{-2}$.

After 6 months, patients in the MD group showed a greater adherence to the MD pattern than those in the PD group (table 2). More specifically, the change in MedDietScore was $12.7 \pm 4.9$ in the MD group and $0.95 \pm 4.5$ in the PD group $(\mathrm{p}<0.001)$. Apart from MedDietScore, patients in the MD group also improved their level of physical activity (metabolic equivalent task (MET) $\mathrm{min} \cdot$ week $^{-1}$ ) more than those in the PD group $(\mathrm{p}<0.05)$. There was also a statistically significant difference between the two study groups regarding the changes in several anthropometric indices. Patients in the MD group showed a greater decrease in WC $(-8.7 \pm 3.6 \mathrm{~cm})$, WC/height ratio $\left(-0.04 \pm 0.02 \mathrm{~cm} \cdot \mathrm{m}^{-1}\right)$ and WC/hip ratio $\left(-0.04 \pm 0.03 \mathrm{~cm} \cdot \mathrm{cm}^{-1}\right)$ than those in the PD group $\left(-5.7 \pm 3.8 \mathrm{~cm},-0.03 \pm 0.02 \mathrm{~cm} \cdot \mathrm{m}^{-1}\right.$ and $-0.02 \pm 0.02 \mathrm{~cm} \cdot \mathrm{cm}^{-1}$, respectively; $\left.\mathrm{p}<0.05\right)$, while there was no difference between the two groups regarding the change in $\mathrm{NC}$ and $\mathrm{NC} /$ height ratio ( $\mathrm{p}>0.05)$. Body fat also decreased more in the MD group than in the PD group $(-4.3 \pm 2.5$ versus $-2.6 \pm 1.7 \% ; \mathrm{p}<0.05)$. At the end of the intervention, although patients in the MD group had reduced their weight and BMI more than those in the PD group $(-8.9 \pm 3.9$ versus $-7.2 \pm 4.2 \mathrm{~kg}$ and $-3.2 \pm 1.5$ versus $-2.5 \pm 1.4 \mathrm{~kg} \cdot \mathrm{m}^{-2}$, respectively), these changes did not reach statistical significance $(p>0.05)$. There were no significant differences in the changes in sleep parameters between the two groups ( $p>0.05)$, except the AHI during REM sleep. AHI/REM improved significantly more in participants in the MD group than in those in the PD group. Specifically, AHI/REM decreased by $18.4 \pm 17.6$ in the MD 
TABLE 1 Patient characteristics at baseline

\begin{tabular}{|c|c|c|c|}
\hline & PD & MD & $\mathrm{p}$-value ${ }^{\#}$ \\
\hline Patients $\mathrm{n}$ & 20 & 20 & \\
\hline Males/females $n$ & $17 / 3$ & $17 / 3$ & 1 \\
\hline Age yrs & $45.8 \pm 14.2$ & $52.2 \pm 10.5$ & 0.09 \\
\hline Educational level & $2.9 \pm 1.1$ & $2.6 \pm 1.2$ & 0.41 \\
\hline Current smoking $\mathrm{n}(\%)$ & $6(30)$ & $6(30)$ & 1 \\
\hline MedDietScore & $28.1 \pm 4.1$ & $28.9 \pm 4.2$ & 0.5 \\
\hline MET min. week $^{-1}$ & $1457.3 \pm 1391.9$ & $1390.9 \pm 1393.9$ & 0.5 \\
\hline Height $\mathbf{m}$ & $1.68 \pm 0.09$ & $1.69 \pm 0.06$ & 0.7 \\
\hline Weight kg & $108.9 \pm 19.8$ & $101.5 \pm 12.5$ & 0.2 \\
\hline BMI $\mathbf{k g} \cdot \mathbf{m}^{-2}$ & $37.7 \pm 4.6$ & $35.3 \pm 3.8$ & 0.04 \\
\hline$W C \mathrm{~cm}$ & $119.3 \pm 14.4$ & $115.6 \pm 9.3$ & 0.3 \\
\hline WC/height ratio $\mathrm{cm} \cdot \mathrm{m}^{-1}$ & $0.7 \pm 0.06$ & $0.67 \pm 0.05$ & 0.09 \\
\hline WC/hip ratio $\mathrm{cm} \cdot \mathrm{cm}^{-1}$ & $0.98 \pm 0.07$ & $1 \pm 0.06$ & 0.4 \\
\hline $\mathrm{NC} \mathrm{cm}$ & $44.3 \pm 4.1$ & $43.4 \pm 3.8$ & 0.5 \\
\hline $\mathrm{NC} /$ height ratio $\mathrm{cm} \cdot \mathrm{m}^{-1}$ & $26.2 \pm 2.02$ & $25.6 \pm 2.2$ & 0.4 \\
\hline BF $^{\pi} \%$ & $32.7 \pm 5.5$ & $34.1 \pm 3.7$ & 0.4 \\
\hline Sleep efficiency \% & $70.3 \pm 15.01$ & $66.3 \pm 15.9$ & 0.4 \\
\hline NREM \% TST & $87.9 \pm 19.8$ & $89.9 \pm 12.5$ & 0.7 \\
\hline SWS \% TST & $6.7 \pm 4.5$ & $6.7 \pm 5.8$ & 1 \\
\hline REM \% TST & $7.7 \pm 3.4$ & $5.4 \pm 3.7$ & 0.06 \\
\hline$A H I$ events $\cdot h^{-1}$ & $58.7 \pm 34.9$ & $52.4 \pm 32.8$ & 0.6 \\
\hline AHI/REM events $\cdot h^{-1}$ & $56.4 \pm 36.7$ & $67.9 \pm 26.2$ & 0.3 \\
\hline Al events $\cdot h^{-1}$ & $52.1 \pm 20.3$ & $56.5 \pm 20.4$ & 0.5 \\
\hline Desaturations per $h$ & $56.9 \pm 33.7$ & $49.4 \pm 29.9$ & 0.5 \\
\hline \multicolumn{4}{|l|}{$\mathrm{Sa}, \mathrm{O}_{2} \%$} \\
\hline Mean & $92.1 \pm 2.9$ & $92.1 \pm 2.7$ & 1 \\
\hline Lowest & $78.2 \pm 8.4$ & $77.4 \pm 6.9$ & 0.7 \\
\hline
\end{tabular}

Data are presented as mean $\pm S D$, unless otherwise stated. Bold indicates statistically significant p-values. PD: prudent diet; MD: Mediterranean diet; MET: metabolic equivalent of task; BMI: body mass index; WC: waist circumference; NC: neck circumference; BF: body fat; NREM: non-rapid eye movement; TST total sleep time; SWS: slow-wave sleep; REM: rapid eye movement; $\mathrm{AHI}$ apnoea-hypopnoea index; $\mathrm{Al}$ : arousal index; $\mathrm{Sa}_{1} \mathrm{O}_{2}$ : arterial oxygen saturation. \#: unpaired t-test; ": four-skinfold thickness method.

\begin{tabular}{|c|c|c|c|}
\hline \multirow[t]{2}{*}{ TABLE 2} & charact & s after $6-m$ & \multirow[b]{2}{*}{$p$-value ${ }^{\#}$} \\
\hline & PD & MD & \\
\hline Patients n & 20 & 20 & \\
\hline MedDietScore & $0.95 \pm 4.5$ & $12.7 \pm 4.9$ & $<0.001$ \\
\hline MET $\min \cdot$ week $^{-1}$ & $47.3 \pm 934.9$ & $369.3 \pm 672.4$ & 0.014 \\
\hline Weight kg & $-7.2 \pm 4.2$ & $-8.9 \pm 3.9$ & 0.162 \\
\hline $\mathrm{BMI} \mathbf{k g} \cdot \mathrm{m}^{-2}$ & $-2.5 \pm 1.4$ & $-3.2 \pm 1.5$ & 0.102 \\
\hline$W C \mathrm{~cm}$ & $-5.7 \pm 3.8$ & $-8.7 \pm 3.6$ & 0.013 \\
\hline WC/height ratio $\mathrm{cm} \cdot \mathrm{m}^{-1}$ & $-0.03 \pm 0.02$ & $-0.04 \pm 0.02$ & 0.044 \\
\hline WC/hip ratio $\mathrm{cm} \cdot \mathrm{cm}^{-1}$ & $-0.02 \pm 0.02$ & $-0.04 \pm 0.03$ & 0.045 \\
\hline $\mathrm{NC} \mathrm{cm}$ & $-0.26 \pm 6.05$ & $-2.9 \pm 5.4$ & 0.169 \\
\hline $\mathrm{NC} /$ height ratio $\mathrm{cm} \cdot \mathrm{m}^{-1}$ & $-0.26 \pm 3$ & $-1.6 \pm 2.6$ & 0.152 \\
\hline BF ${ }^{\mathbb{T}} \%$ & $-2.6 \pm 1.7$ & $-4.3 \pm 2.5$ & 0.032 \\
\hline Sleep efficiency \% & $71.7 \pm 13.8$ & $72.7 \pm 12.4$ & 0.8 \\
\hline NREM \% TST & $90.6 \pm 4.2$ & $92 \pm 5.6$ & 0.4 \\
\hline SWS \% TST & $7.8 \pm 4.2$ & $6.8 \pm 3.6$ & 0.4 \\
\hline REM \% TST & $9.5 \pm 4.8$ & $7.3 \pm 5.6$ & 0.2 \\
\hline AHI events $\cdot h^{-1}$ & $-14 \pm 22.6$ & $-15.6 \pm 11.4$ & 0.791 \\
\hline AHI/REM events $\cdot h^{-1}$ & $-2.6 \pm 23.7$ & $-18.4 \pm 17.6$ & 0.025 \\
\hline Al events $\cdot h^{-1}$ & $-10.2 \pm 19.3$ & $-12.1 \pm 24.9$ & 0.787 \\
\hline Desaturations per $\mathrm{h}$ & $-13.5 \pm 22.6$ & $-13.1 \pm 9.8$ & 0.933 \\
\hline \multicolumn{4}{|l|}{$\mathrm{Sa}, \mathrm{O}_{2} \%$} \\
\hline Mean & $1 \pm 1.5$ & $1.5 \pm 1.7$ & 0.379 \\
\hline Lowest & $3.1 \pm 3.9$ & $4.9 \pm 3.8$ & 0.163 \\
\hline \multicolumn{4}{|l|}{ CPAP use for the } \\
\hline h per day & $6.1 \pm 1.1$ & $6.3 \pm 1.3$ & 0.66 \\
\hline Days per week & $6.4 \pm 0.7$ & $6.4 \pm 0.7$ & 0.75 \\
\hline
\end{tabular}

Data are presented as mean $\pm \mathrm{SD}$, unless otherwise stated. Bold indicates statistically significant p-values. PD: prudent diet; MD: Mediterranean diet; MET: metabolic equivalent of task; BMI: body mass index; WC: waist circumference NC: neck circumference; BF: body fat; NREM; non-rapid eye movement; TST: total sleep time; SWS: slow-wave sleep; REM: rapid eye movement; $\mathrm{AHI}$ : apnoea-hypopnoea index; Al: arousal index; $\mathrm{Sa}_{1} \mathrm{O}_{2}$ : arterial oxygen saturation; CPAP: continuous positive airway pressure. ${ }^{*}$ : unpaired t-test; " : four-skinfold thickness method.

receiving counselling to increase their physical activity. Weight loss trials including both diet and exercise in their programme have the potential to deliver significant health benefits in terms of treating OSAS in the majority of patients with mild [12] and mild-to-moderate [13] OSAS. According to the findings of this study, the MD diet combined with physical activity for a 6month period of time did not lead to a greater improvement in overall OSAS severity than the PD diet, despite better exercise programme compliance. Participants in the MD group demonstrated a greater decrease in WC, WC/height ratio and WC/ hip ratio than the PD group. This means that individuals succeeded in decreasing their abdominal fat. It has been reported that abdominal fat, BMI and NC are significant clinical predictors of OSAS [26, 27]. NC has been suggested to be more predictive of OSAS than general obesity and the lack of impact on overall OSAS severity could be related to lack of significant difference in the change in NC [28]. The only sleep parameter with a remarkable reduction was AHI/REM in 


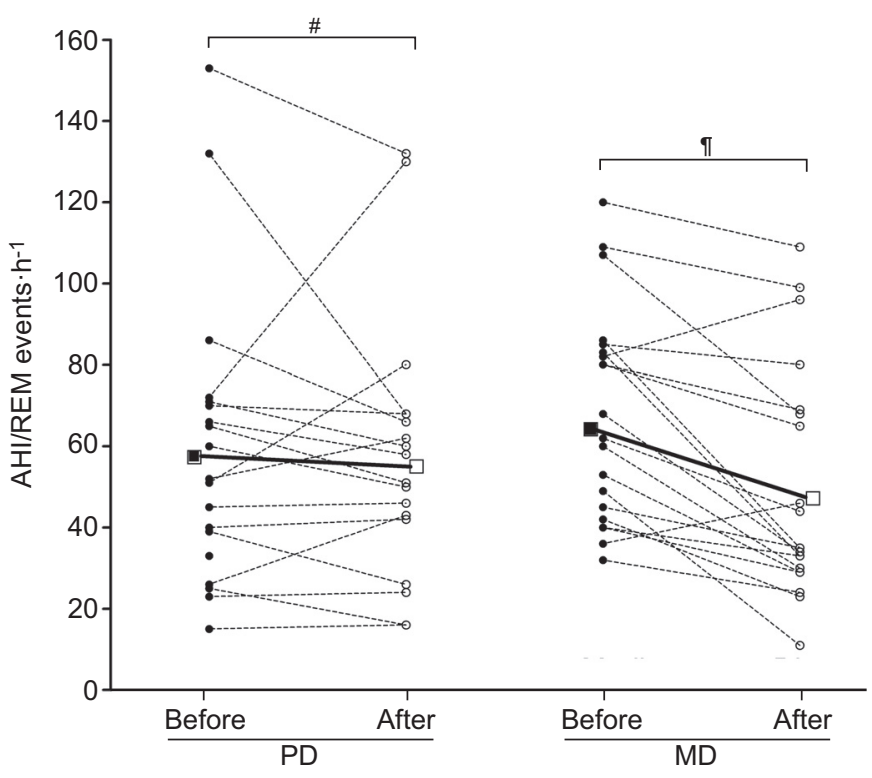

FIGURE 2. Individual values of apnoea-hypopnoea index (AHI) during rapid eye movement (REM) sleep before (closed circles) and after (open circles) 6 months in the prudent diet (PD) versus the Mediterranean diet (MD) group. Squares and solid lines represent mean values. ${ }^{*}: p=0.6 ;{ }^{\circ}: p=0.002$.

patients in the MD arm compared with those following a PD. The finding of AHI/REM was not a primary end-point and further studies are needed in order to clarify this point. This was independent of the baseline value of AHI/REM. This is an interesting finding, since REM sleep abnormalities are associated with decreased upper airway muscle activation $[29,30]$, impaired genioglossus reflex responsiveness to negative pressure [31] and reduced chemosensitivity [32]. All these factors are related to the severity of the syndrome [33]. Recent reports have also related an increased AHI/REM with the risk of developing significant systemic consequences. MAHMOOD et al. [34] recently reported that an increased prevalence of diabetes type II was related to this specific phenotype of OSAS. However, its clinical significance remains unclear, although compared with non-REM sleep, during REM sleep, there is an increase in the duration of apnoea episodes, which perhaps cause more severe desaturation events [35]. There is no evidence from previous weight loss trials using conventional diet programmes to support this finding. This significant difference in the change in AHI/REM between the treatment groups could be related to the notable difference in the change in relevant body circumference indices. It is possible that the MD, along with increased physical activity, could promote changes of relevant anthropometric determinants, such as waist size, and, finally, lead to a better outcome. However, more studies dealing with this specific research question are needed to clarify this point. It has been suggested that abdominal fat is a risk factor for OSAS in obese patients [36]. It is related to a reduction in pharyngeal lumen size due to fatty tissue within the airway or in its lateral walls, and a decrease in upper airway muscle protective force due to fatty deposits in the muscle. It has also been reported that abdominal fat is associated with reduced upper airway size secondary to the mass effect of the large abdomen on the chest wall and tracheal traction [37]. Moreover, an association between increased BMI and the development of hypoventilation is well recognised, as abdominal fat accumulation is known to impair diaphragmatic excursion and rib cage expansion [38]. Since the diaphragm is the main inspiratory muscle during REM sleep [39], abdominal obesity may mechanically affect the expansion of the diaphragm, probably by encroaching into the chest by the chest wall or diaphragm, or by impeding the descent of the diaphragm during forced inspiration [40]. While obesity alone does not account for the development of hypoventilation, it is possible that by decreasing abdominal fat, breathing would be improved during REM sleep. Therefore, it is likely that the patients in the MD group reduced $\mathrm{AHI} / \mathrm{REM}$ more than those in the PD group because of the greater reduction in abdominal fat, via an improvement in the mechanical load associated with OSAS [41]. The greater decrease in body fat in the MD group, as estimated by the fourskinfold method, could also be considered to strengthen our results, since body fat measured in this way has been considered a significant predictor for OSAS [42]. The difference between the two treatment groups in the changes in the above anthropometric indices and in body fat could be attributed to the greater adherence to the prescribed diet and level of physical activity in the patients in the MD group. It has been shown that exercise training alone is not an adequate intervention strategy for most individuals with OSAS but may serve well as an adjunct treatment strategy in the conservative management of OSAS. The results could be attributed to the combination of greater adherence to the hypocaloric diet and exercise in the MD group. Additionally, several reports have shown that abdominal obesity is substantially reduced when combining the above lifestyle modifications [43-45].

Potential mechanisms linking MD adherence to better compliance with a weight loss dietary programme compared to the PD used in this study may include its lower energy density [46] and its relatively low glycaemic load [47]. These beneficial factors, along with its higher fibre [46] and water [48] content, lead to increased satiation and lower calorie intakes. Moreover, the MD is highly palatable, which can increase both tolerance and compliance among individuals following it [49].

Regarding physical activity, there was a higher adoption of counselling by patients in the MD group. This observation was independent of factors such as age (older age), sex (female sex), educational level (low educational level) [50] and smoking [51], which could interfere with the participants' ability to become physically active. These factors did not differ between the two groups at baseline. Recently, it was suggested that an increment in physical activity in conjunction with a healthy diet would lead to optimum weight loss [52]. However, in the present study, no difference in weight loss was observed between the two groups.

A strength of the present study was that participants in both groups were $100 \%$ compliant with CPAP and, therefore, the differences in weight loss and dietary adherence between the groups could not be attributed to CPAP. As the patients knew that their compliance was being evaluated and represented one of the end-points of the study, this may have significantly increased their motivation. Moreover, the physician responsible 
for the patients encouraged and reminded them during the study of the health risks cause by not using CPAP. However, a limitation of the study was the small size of the sample studied. This could be attributed to the characteristics of the population, the rigorous exclusion criteria and the lack of willingness of 23 patients to participate. The duration of the study was also relatively short, given that it has been shown that weight regain is common after weight loss [53], and weight loss programmes should last $\geqslant 1$ yr. However, it has been proposed that the MD pattern can protect against weight gain [54]. All these limitations could adversely affect the external validity of our study and the results should be viewed with great caution. Moreover, the clinical application of our data may be valid only for patients with similar characteristics to those in the population studied.

In conclusion, this randomised weight loss trial showed that the MD combined with physical activity for 6 months was effective in reducing AHI/REM without any statistically significant effect on other sleep parameters, compared with a PD, in obese adults with moderate-to-severe OSAS. However, in view of the beneficial role of the MD and physical activity, further studies with more patients and potentially longer intervention periods will be needed in order to clarify the role of $\mathrm{MD}$ in the therapeutic plan of these patients and to address the potential mechanisms.

\section{CLINICAL TRIAL}

This study is registered at ClinicalTrials.gov with identifier number NCT01312558.

\section{STATEMENT OF INTEREST}

None declared.

\section{ACKNOWLEDGEMENTS}

We would like to acknowledge the help of our colleagues: V. Moniaki and E. Mavroudi (Dept of Thoracic Medicine, Sleep Disorders Unit, University General Hospital, Medical School of the University of Crete, Heraklion, Greece).

\section{REFERENCES}

1 Young T, Palta M, Dempsey J, et al. The occurrence of sleepdisordered breathing among middle-aged adults. $N$ Engl J Med 1993; 328: 1230-1235.

2 Kyzer S, Charuzi I. Obstructive sleep apnea in the obese. World J Surg 1998; 22: 998-1001.

3 Marin JM, Carrizo SJ, Vicente E, et al. Long-term cardiovascular outcomes in men with obstructive sleep apnoea-hypopnoea with or without treatment with continuous positive airway pressure: an observational study. Lancet 2005; 365: 1046-1053.

4 Yim-Yeh S, Rahangdale S, Nguyen AT, et al. Vascular dysfunction in obstructive sleep apnea and type 2 diabetes mellitus. Obesity 2011; 19: 17-22.

5 Freedman N. Treatment of obstructive sleep apnea syndrome. Clin Chest Med 2010; 31: 187-201.

6 Hirshkowitz M, Sharafkhaneh A. Positive airway pressure therapy of OSA. Semin Respir Crit Care Med 2005; 26: 68-79.

7 Jenkinson C, Davies RJ, Mullins R, et al. Comparison of therapeutic and subtherapeutic nasal continuous positive airway pressure for obstructive sleep apnoea: a randomised prospective parallel trial. Lancet 1999; 353: 2100-2105.
8 Hertegonne K, Bauters F. The value of auto-adjustable CPAP devices in pressure titration and treatment of patients with obstructive sleep apnea syndrome. Sleep Med Rev 2010; 14: 115-119.

9 Kajaste S, Brander PE, Telakivi T, et al. A cognitive-behavioral weight reduction program in the treatment of obstructive sleep apnea syndrome with or without initial nasal CPAP: a randomized study. Sleep Med 2004; 5: 125-131.

10 Kansanen M, Vanninen E, Tuunainen A, et al. The effect of a very low-calorie diet-induced weight loss on the severity of obstructive sleep apnoea and autonomic nervous function in obese patients with obstructive sleep apnoea syndrome. Clin Physiol 1998; 4: 377-385.

11 Johansson K, Neovius M, Lagerros YT, et al. Effect of a very low energy diet on moderate and severe obstructive sleep apnoea in obese men: a randomised controlled trial. BMJ 2009; 339: b4609.

12 Tuomilehto HP, Seppä JM, Partinen MM, et al. Lifestyle intervention with weight reduction: first-line treatment in mild obstructive sleep apnea. Am J Respir Crit Care Med 2009; 179: 320-327.

13 Barnes M, Goldsworthy UR, Cary BA, et al. A diet and exercise program to improve clinical outcomes in patients with obstructive sleep apnea - a feasibility study. J Clin Sleep Med 2009; 5: 409-415.

14 Veasey SC, Guilleminault C, Strohl KP, et al. Medical therapy for obstructive sleep apnea: a review by the Medical Therapy for Obstructive Sleep Apnea Task Force of the Standards of Practice Committee of the American Academy of Sleep Medicine. Sleep 2006; 29: 1036-1044.

15 Sweet SN, Fortier MS. Improving physical activity and dietary behaviours with single or multiple health behaviour interventions? A synthesis of meta-analyses and reviews. Int J Environ Res Public Health 2010; 7: 1720-1743.

16 Malik VS, Hu FB. Popular weight-loss diets: from evidence to practice. Nat Clin Pract Cardiovasc Med 2007; 4: 34-41.

17 Schröder H. Protective mechanisms of the Mediterranean diet in obesity and type 2 diabetes. J Nutr Biochem 2007; 18: 149-160.

18 Rechtschaffen A, Kales A, eds. A Manual of Standardized Terminology, Techniques, and Scoring System for Sleep Stages of Human Subjects. Bethesda, US Department of Health, Education and Welfare, 1968.

19 Iber K, Ancoli-Israel S, Chesson AL, et al. The AASM Manual for the Scoring of Sleep and Associated Events. Westchester, American Academy of Sleep Medicine, 200.

20 Pépin JL, Krieger J, Rodenstein D, et al. Effective compliance during the first 3 months of continuous positive airway pressure. A European prospective study of 121 patients. Am J Respir Crit Care Med 1999; 160: 1124-1129.

21 WHO. Obesity: preventing and managing the global epidemic. Report of a WHO Consultation. WHO Technical Report Series 894. http:/ / whqlibdoc.who.int/trs/WHO_TRS_894.pdf Date last accessed: November 29, 2010. Date last updated: 2000.

22 Durnin JV, Womersley J. Body fat assessed from total body density and its estimation from skinfold thickness: measurements on 481 men and women aged from 16 to 72 years. Br J Nutr 1974; 32: 77-97.

23 Papadaki A, Scott JA. The Mediterranean eating in Scotland experience project: evaluation of an Internet-based intervention promoting the Mediterranean diet. Br J Nutr 2005; 94: 290-298.

24 Panagiotakos DB, Pitsavos C, Arvaniti F, et al. Adherence to the Mediterranean food pattern predicts the prevalence of hypertension, hypercholesterolemia, diabetes and obesity, among healthy adults; the accuracy of the MedDietScore. Prev Med 2007; 44: 335-340.

25 Craig CL, Marshall AL, Sjöström M, et al. International physical activity questionnaire: 12 -country reliability and validity. Med Sci Sports Exerc 2003; 35: 1381-1395.

26 Bouloukaki I, Kapsimalis F, Mermigkis C, et al. Prediction of obstructive sleep apnea syndrome in a large Greek population. Sleep Breath 2011; 15: 657-664.

27 Stadler DL, McEvoy RD, Sprecher KE, et al. Abdominal compression increases upper airway collapsibility during sleep in obese male obstructive sleep apnea patients. Sleep 2009; 32: 1579-1587. 
28 Davies RJ, Ali NJ, Stradling JR. Neck circumference and other clinical features in the diagnosis of the obstructive sleep apnoea syndrome. Thorax 1992; 47: 101-105.

29 Horner RL. Motor control of the pharyngeal musculature and implications for the pathogenesis of obstructive sleep apnea. Sleep 1996; 19: 827-853.

30 Wiegand L, Zwillich CW, Wiegand D, et al. Changes in upper airway muscle activation and ventilation during phasic REM sleep in normal men. J Appl Physiol 1991; 71: 488-497.

31 Shea SA, Edwards JK, White DP. Effect of wake-sleep transitions and rapid eye movement sleep on pharyngeal muscle response to negative pressure in humans. J Physiol 1999; 520: 897-908.

32 Douglas NJ, White DP, Weil JV, et al. Hypoxic ventilatory response decreases during sleep in normal men. Am Rev Respir Dis 1982; 125: 286-289.

33 Younes M. Contributions of upper airway mechanics and control mechanisms to severity of obstructive apnea. Am J Respir Crit Care Med 2003; 168: 645-658.

34 Mahmood K, Akhter N, Eldeirawi K, et al. Prevalence of type 2 diabetes in patients with obstructive sleep apnea in a multi-ethnic sample. J Clin Sleep Med 2009; 5: 215-221.

35 Findley LJ, Wilhoit SC, Suratt PM. Apnea duration and hypoxemia during REM sleep in patients with obstructive sleep apnea. Chest 1985; 87: 432-436.

36 Ucok K, Aycicek A, Sezer M, et al. Aerobic and anaerobic exercise capacities in obstructive sleep apnea and associations with subcutaneous fat distributions. Lung 2009; 187: 29-36.

37 Seidell JC. Waist circumference and waist/hip ratio in relation to all-cause mortality, cancer and sleep apnea. Eur J Clin Nutr 2010; 64: 35-41.

38 Schäfer H, Pauleit D, Sudhop T, et al. Body fat distribution, serum leptin, and cardiovascular risk factors in men with obstructive sleep apnea. Chest 2002; 122: 829-839.

39 Bennett JR, Dunroy HM, Corfield DR, et al. Respiratory muscle activity during REM sleep in patients with diaphragm paralysis. Neurology 2004; 62: 134-137.

40 Lazarus R, Gore CJ, Booth M, et al. Effects of body composition and fat distribution on ventilatory function in adults. Am J Clin Nutr 1998; 68: 35-41.

41 Patil SP, Schneider H, Marx JJ, et al. Neuromechanical control of upper airway patency during sleep. J Appl Physiol 2007; 102: 547-556.
42 Casale M, Pappacena M, Rinaldi V, et al. Obstructive sleep apnea syndrome: from phenotype to genetic basis. Curr Genomics 2009; 10: $119-126$.

43 Norman JF, Von Essen SG, Fuchs RH, et al. Exercise training effect on obstructive sleep apnea syndrome. Sleep Res Online 2000; 3: 121-129.

44 Christiansen T, Paulsen SK, Bruun JM, et al. Comparable reduction of the visceral adipose tissue depot after a diet-induced weight loss with or without aerobic exercise in obese subjects: a 12-week randomized intervention study. Eur J Endocrinol 2009; 160: 759-767.

45 Carr DB, Utzschneider KM, Boyko EJ, et al. A reduced-fat diet and aerobic exercise in Japanese Americans with impaired glucose tolerance decreases intra-abdominal fat and improves insulin sensitivity but not beta-cell function. Diabetes 2005; 54: 340-347.

46 Schröder H. Protective mechanisms of the Mediterranean diet in obesity and type 2 diabetes. J Nutr Biochem 2007; 18: 149-160.

47 Brand-Miller J, McMillan-Price J, Steinbeck K, et al. Dietary glycemic index: health implications. J Am Coll Nutr 2009; 28: 446-449.

48 Davy BM, Dennis EA, Dengo AL, et al. Water consumption reduces energy intake at a breakfast meal in obese older adults. J Am Diet Assoc 2008; 108: 1236-1239.

49 Zazpe I, Sanchez-Tainta A, Estruch R, et al. A large randomized individual and group intervention conducted by registered dietitians increased adherence to Mediterranean-type diets: the PREDIMED study. J Am Diet Assoc 2008; 108: 1134-1144.

50 Johansen KL, Chertow GM, Kutner NG, et al. Low level of selfreported physical activity in ambulatory patients new to dialysis. Kidney Int 2010; 78: 1164-1170.

51 Pate RR, Heath GW, Dowda M, et al. Associations between physical activity and other health behaviors in a representative sample of US adolescents. Am J Public Health 1996; 86: 1577-1581.

52 Caudwell P, Hopkins M, King NA, et al. Exercise alone is not enough: weight loss also needs a healthy (Mediterranean) diet? Public Health Nutr 2009; 12: 1663-1666.

53 Franz MJ, VanWormer JJ, Crain AL, et al. Weight-loss outcomes: a systematic review and meta-analysis of weight-loss clinical trials with a minimum 1-year follow-up. J Am Diet Assoc 2007; 107: 1755-1767.

54 Buckland G, Bach A, Serra-Majem L. Obesity and the Mediterranean diet: a systematic review of observational and intervention studies. Obes Rev 2008; 9: 582-593. 